\title{
Molecular genetics of exercise-induced polymorphic ventricular tachycardia: identification of three novel cardiac ryanodine receptor mutations and two common calsequestrin 2 amino-acid polymorphisms
}

\author{
Päivi J Laitinen ${ }^{1}$, Heikki Swan ${ }^{2}$ and Kimmo Kontula*,1 \\ ${ }^{1}$ Department of Medicine and Biomedicum Helsinki, Helsinki, Finland; ${ }^{2}$ Department of Cardiology, University of \\ Helsinki, Helsinki, Finland
}

Mutations of two myocardial calcium signaling molecules, ryanodine receptor 2 (RYR2) and calsequestrin 2 (CASQ2), may cause catecholaminergic polymorphic ventricular tachycardia (CPVT), a severe inherited arrhythmic disease manifesting with salvoes of exercise-induced bidirectional and polymorphic tachycardias. We screened 12 Finnish CPVT probands for mutations in these genes and identified three novel RYR2 mutations (V2306I, P4902L, R4959Q), which were absent in unaffected and control individuals. Although no obvious disease-causing mutations were identified in the CASQ2 gene, the molecular screening revealed two novel amino-acid polymorphisms (T66A and V76M). The frequencies of these polymorphisms in 185 unrelated probands with long QT syndrome and in $\mathbf{2 8 0}$ healthy blood donors were not significantly different. These data, combined with our previous findings, show that RYR2 mutations are present in at least $6 / 16(38 \%)$ of the catecholaminergic polymorphic ventricular tachycardia families, while CASQ2 mutations must be a rare cause of CPVT.

European Journal of Human Genetics (2003) 11, 888-891. doi:10.1038/sj.ejhg.5201061

Keywords: ventricular arrhythmia; mutation; ryanodine receptor; polymorphism; calsequestrin

\section{Introduction}

Familial polymorphic ventricular tachycardia (FPVT, recently assigned as catecholaminergic polymorphic ventricular tachycardia, CPVT) (OMIM \#604772) is a rare autosomal dominantly inherited disorder characterized by episodes of polymorphic or bidirectional ventricular tachycardias and risk of sudden death. Physical exercise and adrenergic stimulation typically trigger the symptoms, while no structural abnormalities of the heart can be observed. We assigned the disease locus to chromosome $1 \mathrm{q} 42-\mathrm{q} 43$. $^{1}$ Soon thereafter, we and others showed that mutations in the gene encoding the myocardial calcium

*Correspondence: Dr K Kontula, Department of Medicine, University of Helsinki, Haartmaninkatu 4, FIN-00290, Helsinki, Finland. Tel: 35894717 2230; Fax: 35894717 4013; E-mail: kimmo.kontula@hus.fi Received 18 March 2003; revised 2 June 2003; accepted 10 June 2003 release channel ryanodine receptor (RYR2) cause CPVT. ${ }^{2,3}$ RYR2 mutations are also responsible for a variant form of arrhythmogenic right ventricular dysplasia (ARVD2). ${ }^{4}$ To date, altogether 21 RYR2 mutations have been reported. ${ }^{2-6}$ Lahat et $\mathrm{al}^{7}$ described the recessive form of the disease, localized the disease-causing gene to chromosome $1 \mathrm{p} 13-21,{ }^{7}$ and subsequently identified a missense mutation in the calsequestrin 2 gene (CASQ2). ${ }^{8}$

RYR2 and CASQ2 form a complex with junctin and triadin at the junctional sarcoplasmic reticulum (SR) membrane $e^{9-11}$ and are critically involved in cardiac excitation-contraction coupling. Upon depolarization of the plasma membrane, elevation of the cytosolic calcium concentration induces RYR2 channels to open and release $\mathrm{Ca}^{2+}$ from the SR, a mechanism termed calcium-induced calcium release. ${ }^{12}$ Calsequestrins bind large amounts of $\mathrm{Ca}^{2+}$, thereby lowering the level of free $\mathrm{Ca}^{2+}$ within the 
lumen of SR and inhibiting intrasarcoplasmic precipitation of $\mathrm{Ca}^{2+}$ ions. ${ }^{13}$

The aim of this study was to clarify whether mutations of the CASQ2 gene could underlie the dominant form of CPVT in Finnish families. In addition, we conducted a search for novel RYR2 mutations in the same families.

\section{Materials and methods}

\section{Patients and controls}

Diagnostic criteria for CPVT were described previously. ${ }^{1,14}$ In short, resting ECG in these patients was normal, and cardiac imaging studies did not demonstrate any evidence of structural alterations of the heart. The patients had frequent ventricular premature complexes or ventricular tachycardia during exercise stress test, which is the most sensitive diagnostic test for CPVT. Recently, we screened the whole coding region of the RYR2 gene in the samples of four CPVT probands (group 1) and identified three missense mutations. ${ }^{2}$ In the present study, we searched for RYR2 mutations in the samples of 12 unrelated Finnish CPVT probands (group 2) whose DNA had not been analyzed previously. While the screening of RYR2 gene was in progress, clinical studies revealed seven new families (group 3) affected with CPVT. Samples of groups 2 and 3 $(n=19)$ were screened for mutations in CASQ2 gene. Two CASQ2 polymorphisms identified were also determined in a cohort of 185 unrelated probands with long QT syndrome (LQTS), in which previous molecular studies had failed to detect disease-causing mutations. Samples from 280 healthy Finnish blood donors (kindly provided by Dr Tom Krusius, the Finnish Red Cross Blood Service) were used as controls. Informed consent was obtained from all patients and the study was approved by the Ethics Review Committee of the department.

\section{Mutation screening}

In 12 probands, approximately $50 \%$ of the coding portion of RYR2 gene, including the exons 44-47 and 83-105 harboring all the hitherto reported CPVT-causing RYR2 mutations, was amplified by PCR and sequenced. Dr Hadas Lahat (Danek Gartner Institute of Human Genetics) kindly provided the primer sequences for amplification of all 11 CASQ2 exons and for subsequent dHPLC analysis (Wave 3500A equipment, Transgenomic, Omaha, NE, USA). In order to detect possible homozygous alterations, the analysis was repeated with pooled samples containing patient and control PCR products in a ratio of 1:1. All aberrant conformers were sequenced.

Detection of the specific RYR2 mutations was carried out by PCR and restriction analysis. For V2306I and P4902L, artificial recognition sites were generated. The enzymes used were TaaI for V2306I, HaeIII for P4902L, and MspI for R4959Q. CASQ2 T66A was detected by Hin6I and V76M was detected by solid-phase minisequencing ${ }^{15}$ using

DynaZyme II DNA Polymerase (Finnzymes, Espoo, Finland).

\section{Statistical analyses}

Comparisons between the groups were performed with $\chi^{2}$ and Fisher's exact tests. $P$-value of $<0.05$ was considered to be statistically significant.

\section{Results}

\section{Novel RYR2 mutations}

We identified three novel missense mutations in the RYR2 gene (Figure 1). A valine-to-isoleucine change at position 2306 (nucleotide $\mathrm{G} 6916 \rightarrow \mathrm{A}$ ) was detected in a family of 12 members, where the 24-year-old proband and her 4-yearold son carried the mutation (Family 44087, Figure 2). The

a

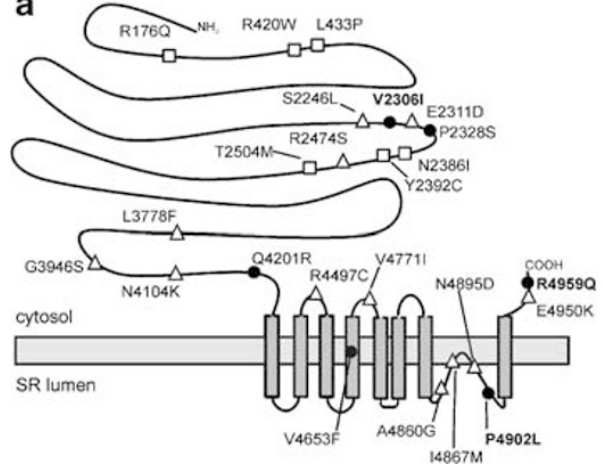

b

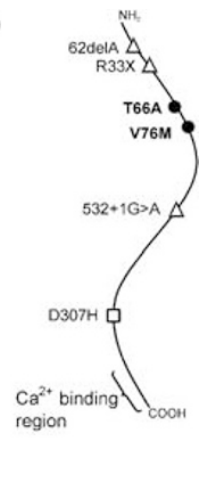

Figure 1 (a) A schematic view of the RYR2 protein (according to the model of Du et $a l^{20}$ ) containing all mutations described thus far. Filled circles denote mutations described in this (bold) and our previous ${ }^{2}$ study, open triangles show mutations described by Priori et $a l^{3,5}$ open squares by Tiso et $a l^{4}$ and Bauce et al. ${ }^{6}$ (b) Approximate location of all the reported CASQ2 mutations by Lahat et $a l^{8}$ (open square) and Postma et $a l^{15}$ (open triangles). The polymorphisms described in this study are denoted with filled circles. The location of the predicted $\mathrm{Ca}^{2+}$-binding region is implicated.

Family 44087

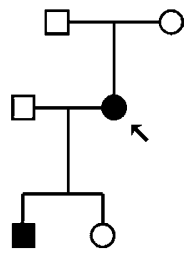

Figure 2 Pedigrees of the three families with identified RYR2 mutations. Filled symbols denote affected individuals, empty symbols denote nonaffected individuals, and slashed symbols denote deceased individuals. Probands are indicated with an arrow. The spouses of the probands were not studied. 
proband had her first syncopal spell under the age of 7 years and several episodes thereafter at the age of 10-13 years. Since then, she has been asymptomatic while on beta-antiadrenergic blocking medication. The V2306I mutation was absent in both the parents and other relatives of the proband. Haplotype analysis confirmed paternity, indicating a de novo type of mutation. A P4902L (nucleotide $\mathrm{C} 14705 \rightarrow \mathrm{T}$ ) mutation was present in a 56-yearold female proband whose parents were deceased. Her clinically unaffected siblings and son did not carry the mutation (Family 54003, Figure 2). The proband has had three syncopal spells after the age of 20 years upon strenuous physical effort such as running. At the extreme $3^{\prime}$ end of the RYR2 gene, we identified a G14876 $\rightarrow$ A alteration, predicted to change arginine 4959 to glutamine (R4959Q), in a female proband (age 60 years) whose three unaffected children were not carriers of this mutation (Family 54151, Figure 2). The proband had experienced syncopal spells twice at the age of 40 years. The parents of the proband were deceased. None of these nucleotide changes was detected in a cohort of 180 healthy Finnish blood donors.

\section{CASQ2 amino-acid polymorphisms}

The DNA sample of a 42-year-old CPVT proband was found to be heterozygous for two nucleotide changes A195 $\rightarrow \mathrm{G}$ and G225 $\rightarrow$ A, which were predicted to result in aminoacid substitutions T66A and V76M (Figure 1), respectively. Both aberrations were present in the same allele as demonstrated by subcloning of the PCR product into a plasmid and its subsequent sequencing. In the family of the proband, his affected son and mother, together with his unaffected daughter, were heterozygous for both T66A and V76M. T66A alone was present in seven other index cases, while V76M could not be detected in any of the other 18 index cases studied. In addition, we detected several intronic nucleotide substitutions (IVS2-77C $>\mathrm{T}$, IVS3 + 6T $>$ C, IVS3-14G $>$ A, IVS7-89C $>$ T, IVS7-29G $>$ A, IVS7-17T $>$ A, IVS9-39C $>$ G, and IVS10-119G $>A$ ), probably representing common polymorphisms. Two silent polymorphisms $(\mathrm{C} 1184 \rightarrow \mathrm{T}$ and $\mathrm{T} 1193 \rightarrow \mathrm{C})$ were found in exon 11 of the CASQ2 gene.

To assess the possible phenotypic effects of T66A and V76M polymorphisms on ventricular repolarization, we compared the frequencies of the two polymorphisms alone and the resulting haplotypes in LQTS probands and healthy controls, and noticed no significant difference (Table 1).

\section{Discussion}

Recently, two disease-causing genes, RYR2 ${ }^{2,3}$ and CASQ2, ${ }^{8}$ were identified to underlie FPVT, a devastating inherited heart disease. The present study provides additional support for the pathophysiologic role of the RYR2 channel
Table 1 Genotype, allele, and haplotype numbers (frequencies in percentages shown in parentheses) for the two CASQ2 polymorphisms

\begin{tabular}{lccc}
\hline Classification & CPVT indexes & LQTS & Controls \\
\hline T66A & & & \\
Genotypes & & & \\
TT & $11(57.8 \%)$ & $101(57.1 \%)$ & $136(51.3 \%)$ \\
AT & $8(42.1 \%)$ & $61(34.5 \%)$ & $107(40.4 \%)$ \\
AA & $0(0 \%)$ & $15(8.5 \%)$ & $22(8.3 \%)$ \\
Alleles & & & \\
Allele T & $30(78.9 \%)$ & $263(74.3 \%)$ & $379(71.5 \%)$ \\
Allele A & $8(21.2 \%)$ & $91(25.7 \%)$ & $151(28.5 \%)$ \\
& & & \\
V76M & & & \\
Genotypes & $18(94.7 \%)$ & $175(94.6 \%)$ & $257(94.8 \%)$ \\
VV & $1(5.3 \%)$ & $10(5.4 \%)$ & $14(5.2 \%)$ \\
VM & $0(0 \%)$ & $0(0 \%)$ & $0(0 \%)$ \\
MM & & & \\
Alleles & $1(97.4 \%)$ & $360(97.3 \%)$ & $528(97.4 \%)$ \\
Allele V & $10(2.7 \%)$ & $14(2.6 \%)$ \\
Allele M & $1(2.6 \%)$ & & \\
Haplotype & & & \\
T66-V76 & $30(78.9 \%)$ & $257(72.6 \%)$ & $364(70.5 \%)$ \\
T66-M76 & $0(0 \%)$ & $0(0 \%)$ & $2(0.4 \%)$ \\
A66-V76 & $7(18.4 \%)$ & $81(22.9 \%)$ & $133(25.8 \%)$ \\
A66-M76 & $1(2.6 \%)$ & $4(1.1 \%)$ & $5(1.0 \%)$ \\
\hline All & &
\end{tabular}

All differences were statistically nonsignificant. ${ }^{a}$ In all, 12 cases (six in the LQTS group and six in the control group) were heterozygous for both loci, rendering exact haplotyping impossible.

in CPVT. Our data show that although CASQ2 has been implemented to cause CPVT in patients from Israel, ${ }^{8}$ France, ${ }^{16}$ and Netherlands, ${ }^{16}$ this gene may play a minor, if any, role in Finnish patients.

The three novel RYR2 mutations (V2306I, P4902L, and R4959Q) occurred in affected members of the three families, but were absent in over 350 control chromosomes. All these mutations alter an evolutionarily conserved amino acid and are situated in areas previously known to harbor CPVT mutations (Figure 1). Screening of that portion of the coding region of RYR2 proposed to be pathophysiologically significant, as well as the total coding region of CASQ2, did not reveal any other significant alterations in these individuals. Therefore, we judged the mutations to be causative in these families in which linkage analyses were hampered by small pedigree size.

Two of the three missense mutations identified in the present study result in amino-acid substitutions in the membrane-associated carboxy-terminus and one in the central portion of the RYR2 protein encompassing the binding region for the regulatory protein FKBP12.6. ${ }^{17}$ Most of the hitherto identified mutations cluster to these two domains that accordingly must contain functionally important regions. The phenotype of the affected individuals identified were characteristic of CPVT, ${ }^{1}$ although the age of onset of the symptoms among the probands varied between 7 and 40 years. The number of patients was too small to permit a detailed analysis of genotype-phenotype relationships between different mutations. 
The original report linking CASQ2 to pathogenesis of CPVT suggested a recessive form of inheritance. ${ }^{8}$ Recently, Postma et $a^{16}$ described three families with CASQ2 mutations, and further suggested that one particular nonsense mutation (R33X) could have been inherited in an autosomal-dominant manner with reduced penetrance. This encouraged us to examine the same gene in 19 Finnish pedigrees, with CPVT showing a dominant or unknown mode of inheritance. Upon screening for the whole protein-coding region of CASQ2, we failed to identify any causative mutations. The two relatively common amino-acid changes identified most likely represent innocent genetic polymorphisms, although exact determination of their functional properties demands more specific experiments. Cardiac calsequestrins bind $\mathrm{Ca}^{2+}$ with high capacity and moderate affinity, binding and releasing 35-40 mol of $\mathrm{Ca}^{2+}$ in each excitationcontraction cycle. ${ }^{18}$ Calsequestrin polymerizes into soluble extended structures, and $\mathrm{Ca}^{2+}$ ions appear to be bound to negatively charged residues located in the C-terminal part of the molecule ${ }^{19}$ (Figure 1).

The fact that 10 out of the 16 probands screened in this and our previous ${ }^{2}$ study lacked mutations in the region screened from RYR2, and none of the 19 probands examined had CASQ2 mutations, suggests that there are yet unidentified CPVT-causing genes. The identification of these genes may be facilitated by structural analysis of other calcium-signaling molecules or by linkage analysis in families with multiple affected individuals.

\section{Acknowledgements}

Ms Saara Nyqvist and Mrs Tuula Soppela are acknowledged for expert technical assistance. This study was financially supported by a grant from the Maud Kuistila Memorial Foundation, The Finnish Foundation for Cardiovascular Reseach, The Sigrid Juselius Foundation, and the Finnish Academy.

\section{References}

1 Swan H, Piippo K, Viitasalo M et al: Arrhythmic disorder mapped to chromosome 1q42-q43 causes malignant polymorphic ventricular tachycardia in structurally normal hearts. J Am Coll Cardiol 1999; 34: 2035-2042.

2 Laitinen PJ, Brown KM, Piippo K et al: Mutations of the cardiac ryanodine receptor (RyR2) gene in familial polymorphic ventricular tachycardia. Circulation 2001; 103: 485-490.

3 Priori SG, Napolitano C, Tiso N et al: Mutations in the cardiac ryanodine receptor gene (hRyR2) underlie catecholaminergic polymorphic ventricular tachycardia. Circulation 2001; 103: $196-200$
4 Tiso N, Stephan DA, Nava A et al: Identification of mutations in the cardiac ryanodine receptor gene in families affected with arrhythmogenic right ventricular cardiomyopathy type 2 (ARVD2). Hum Mol Genet 2001; 10: 189-194.

5 Priori SG, Napolitano C, Memmi M et al: Clinical and molecular characterization of patients with catecholaminergic polymorphic ventricular tachycardia. Circulation 2002; 106: 69-74.

6 Bauce B, Rampazzo A, Basso C et al: Screening for ryanodine receptor type 2 mutations in families with effort-induced polymorphic ventricular arrhythmias and sudden death: early diagnosis of asymptomatic carriers. J Am Coll Cardiol 2002; 40: $341-349$.

7 Lahat H, Eldar M, Levy-Nissenbaum E et al: Autosomal recessive catecholamine- or exercise-induced polymorphic ventricular tachycardia: clinical features and assignment of the disease gene to chromosome 1p13-21. Circulation 2001; 103: $2822-2827$.

8 Lahat $\mathrm{H}$, Pras E, Olender T et al: A missense mutation in a highly conserved region of CASQ2 is associated with autosomal recessive catecholamine-induced polymorphic ventricular tachycardia in Bedouin families from Israel. Am J Hum Genet 2001; 69: $1378-1384$

9 Zhang L, Kelley J, Schmeisser G, Kobayashi YM, Jones LR: Complex formation between junctin, triadin, calsequestrin, and the ryanodine receptor. Proteins of the cardiac junctional sarcoplasmic reticulum membrane. I Biol Chem 1997; 272: 23389-23397.

10 Guo W, Campbell KP: Association of triadin with the ryanodine receptor and calsequestrin in the lumen of the sarcoplasmic reticulum. J Biol Chem 1995; 270: 9027-9030.

11 Tunwell RE, Wickenden C, Bertrand BM et al: The human cardiac muscle ryanodine receptor-calcium release channel: identification, primary structure and topological analysis. Biochem $J$ 1996; 318: 477-487.

12 Bers DM: Cardiac excitation-contraction coupling. Nature 2002; 415: 198-205.

13 Slupsky JR, Ohnishi M, Carpenter MR, Reithmeier RA: Characterization of cardiac calsequestrin. Biochemistry 1987; 26: 6539-6544.

14 Swan H, Laitinen PJ: Familial polymorphic ventricular tachycardia - intracellular calcium channel disorder. Card Electrophysiol Rev 2002; 6: 81-87.

15 Syvanen AC, Aalto-Setala K, Harju L, Kontula K, Soderlund H: A primer-guided nucleotide incorporation assay in the genotyping of apolipoprotein E. Genomics 1990; 8: 684-692.

16 Postma AV, Denjoy I, Hoorntje TM et al: Absence of calsequestrin 2 causes severe forms of catecholaminergic polymorphic ventricular tachycardia. Circ Res 2002; 91: e21-e26.

17 Marx SO, Reiken S, Hisamatsu Y et al: PKA phosphorylation dissociates FKBP1.6 from the calcium release channel (ryanodine receptor): defective regulation in failing hearts. Cell 2000; 101: $365-376$.

18 Mitchell RD, Simmerman HK, Jones LR: $\mathrm{Ca}^{2+}$ binding effects on protein conformation and protein interactions of canine cardiac calsequestrin. J Biol Chem 1988; 263: 1376-1381.

19 MacLennan DH, Reithmeier RA: Ion tamers. Nat Struct Biol 1998; 5: 409-411.

20 Du GG, Sandhu B, Khanna VK, Guo XH, MacLennan DH: Topology of the $\mathrm{Ca}^{2+}$ release channel of skeletal muscle sarcoplasmic reticulum (RyR1). Proc Natl Acad Sci USA 2002; 99: $16725-16730$. 